\title{
Principais queixas osteomusculares em músicos da região do $A B C$ paulista: um estudo de prevalência
}

\author{
Main musculoskeletal complaints by musicians in \\ the ABC Paulista region: a prevalence study
}

Nádia Reijani ${ }^{1}$, Fernanda Antico Benetti ${ }^{2}$

${ }^{1}$ Curso de Fisioterapia da Faculdade de Medicina do ABC (FMABC) - Santo André (SP), Brasil.

${ }^{2}$ Disciplina de Fisioterapia Preventiva, FMABC - Santo André (SP), Brasil.

DOI: http://dx.doi.org/10.7322/abcshs.v41i1.844

\begin{abstract}
RESUMO
Introdução: Os músicos são profissionais com diversos fatores predisponentes a desenvolver $\mathrm{O}$ adoecimento ocupacional. $\mathrm{Na}$ maioria das vezes, eles apresentam queixas graves. A conscientização do músico para os riscos que ele pode sofrer e os métodos de prevenção são essenciais para evitar maiores danos que possam impedir a prática da atividade que o profissional exerce, uma vez que a música é relacionada ao bem-estar, ao equilíbrio emocional e ao divertimento. Objetivo: Levantar dados e analisar quais são as principais queixas osteomusculares dos músicos devido à postura adquirida na prática instrumentista. Métodos: $\mathrm{A}$ amostra conteve 80 músicos estudantes e profissionais da Associação Cultural Tocando a Vida, da Big Band da Fundação das Artes de São Caetano do Sul e da Orquestra Sinfônica Jovem da Fundação das Artes de São Caetano do Sul. Esses músicos passaram por uma triagem e posteriormente responderam o Questionário Nórdico de Sintomas Osteomusculares. Resultados: Foi observado que, para todas as famílias de instrumentos, a metade ou mais apresentam dor causada pela prática instrumentista. Conclusão: Pode-se concluir que as regiões das mãos e da coluna tanto dorsal quanto lombar foram os locais mais afetados pela prática instrumentista independente de a qual família o instrumento pertença. Não foi possível analisar e relacionar a prevalência das lesões osteomusculares dos músicos, pois a grande maioria não realizava acompanhamento médico e não apresentava diagnóstico clínico.
\end{abstract}

Palavras-chave: LER-DORT; lesões; reabilitação; prevenção; fisioterapia.

\begin{abstract}
Introduction: Musicians are professionals with several predisposing factors to developing the occupational illness. Most of the time, they present serious complaints. Awareness of the musician to the risks that he may suffer and methods of prevention are essential to prevent further damage that might prevent the practice of activity that the trader carries, since music is related to well-being, emotional balance and fun. Objective: Collect data and analyze what are the main musculoskeletal complaints of musicians due to posture acquired instrumentalist practice. Methods: The sample contained 80 musicians, students and professionals of the Associação Cultural Tocando a Vida, Big Band da Fundação das Artes de São Caetano do Sul and the Orquestra Sinfônica da Fundação das Artes de São Caetano do Sul. These musicians have gone through a screening and after answered the questionnaire Nordic Musculoskeletal. Results: It was observed that for all the instruments of half or more families have pain caused by the practice instrumentistal. Conclusion: It can be concluded that the regions of hands and lumbar spine were the places most affected by the practice of instruments. It was not possible to analyze and relate the prevalence of musculoskeletal injuries on the musicians, because the vast majority did not perform medical care and had no clinical diagnosis
\end{abstract}

Keywords: cumulative trauma disorders; injuries; rehabilitation; prevention; physical therapy specialty.

Recebido em: 02/05/2015

Revisado em: 15/10/2015

Aprovado em: 16/12/2015

Autor para correspondência: Fernanda Antico Benetti - Faculdade de Medicina do ABC - Avenida Príncipe de Gales, 821 - Príncipe de Gales CEP: 09060-650 - Santo André (SP) - E-mail: benetti.fernanda@gmail.com

Conflito de interesses: nada a declarar. 


\section{INTRODUÇÃO}

A saúde muscular do músico é um assunto pouco discutido na ciência médica e que precisa de bastante atenção. Os músicos são profissionais que têm diversos fatores predisponentes ao surgimento de adoecimento ocupacional ${ }^{1,2}$, entre eles a forma e o tamanho do instrumento, a técnica, o tempo de trabalho dedicado ao instrumento, as condições de clima e espaço, o comportamento de estudo e ensaio, as estratégias de compensação e as condições corporais dos músicos ${ }^{3-5}$. Porém, fatores intrínsecos podem estar relacionados, como constituição corporal, estado físico, trofismo, grau de flexibilidade muscular e/ou patologia muscular prévia ${ }^{6}$.

A postura em relação aos instrumentos, em sua maioria, é assimétrica e não ergonômica ${ }^{1,4,7}$. Essa assimetria desenvolve alterações e, consequentemente, dores, muitas vezes ignoradas, pois o afastamento para a recuperação pode implicar na perda de oportunidades no mercado de trabalho ${ }^{1}$. As exigências profissionais, que excedem o limite físico e demandam força excessiva, são outro fator que pode acarretar lesões causadas pelo superuso (overuse), sendo que os tecidos mais atingidos por esse excesso de carga são os músculos, os tendões, as articulações e os ligamentos ${ }^{6}$. O termo overuse é também conhecido como Lesão por Esforço Repetitivo (LER) ou desordem por trauma cumulativo e é usado quando o trabalho está relacionado aos sintomas de fraqueza, perda de controle ou velocidade . $^{8}$

$\mathrm{Na}$ maioria das vezes, os músicos apresentam queixas graves. Podemos citar: dor, formigamento ou dormência, fadiga ou fraqueza muscular, dor de cabeça, "ombro congelado", epicondilite, hérnia de disco, torcicolo, problemas nas Articulações Temporomandibulares (ATMs), distonia focal, problemas nos nervos periféricos, entre outros ${ }^{9}$.

A conscientização do músico para os riscos que ele pode correr é essencial para evitar maiores danos que possam impedir a prática da atividade que o profissional exerce, uma vez que a música é relacionada ao bem-estar, ao equilíbrio emocional e ao divertimento ${ }^{10}$. Para isso, a prevenção pode ser feita de diversas formas: o uso correto da mecânica corporal e postural, a redução na duração do tocar, as pausas de recuperação, a realização de exercícios corporais e alongamentos nos intervalos dos ensaios/ prática e a manutenção de bons hábitos de saúde ${ }^{6}$.

Visando oferecer métodos preventivos para evitar lesões e quadros dolorosos aos músicos, torna-se necessário verificarmos quais são as principais queixas osteomusculares e a prevalências das que são apresentadas por eles.

Portanto, o objetivo deste trabalho é levantar dados e analisar quais são as principais queixas osteomusculares dos músicos devido à postura adquirida na prática instrumentista.

\section{MÉTODOS}

Trata-se de um estudo observacional de prevalência, realizado com 80 músicos estudantes e profissionais da Associação Cultural
Tocando a Vida, da Big Band da Fundação das Artes de São Caetano do Sul e da Orquestra Sinfônica Jovem da Fundação das Artes de São Caetano do Sul - 40 tocam instrumentos de cordas ${ }^{9}$, tocam instrumentos de percussão e 31 tocam instrumentos de sopro. A coleta foi realizada entre maio e agosto de 2014.

Dentre os instrumentos de cordas estão violão, guitarra, baixo elétrico, violino, viola, violoncelo, piano e contrabaixo acústico. Os instrumentos de sopro foram compostos por flauta transversal, clarinete, saxofone alto e barítono, tuba, trombone, trompete e trompa. O instrumento de percussão foi a bateria.

Primeiramente o projeto foi aprovado pelo Comitê de Ética da Faculdade de Medicina do ABC pelo parecer $n^{\circ} 569.980$. Em seguida, os voluntários foram informados da pesquisa e, após, foi entregue o Termo de Consentimento Livre e Esclarecido para que assinassem. Posteriormente, os músicos foram submetidos a uma triagem para que fossem caraterizados de acordo com as seguintes variáveis: idade, sexo, qual instrumento pratica, a frequência de ensaio (horas/semana), frequência de estudo (horas/semana), apresenta dor, realiza acompanhamento médico, apresenta diagnóstico, realiza tratamento e qual o tratamento, se pratica exercício físico e qual exercício físico. Após a triagem, eles preencheram o Questionário Nórdico de Sintomas Osteomusculares.

O Questionário Nórdico de Sintomas Osteomusculares é um questionário composto por quatro itens, aplicado para pescoço, ombros, região da coluna dorsal, cotovelos, punhos/mãos, região da coluna lombar, quadril/coxas, joelhos e tornozelos/ pés, com o intuito de avaliar os distúrbios musculares.

Os critérios de inclusão foram: músicos maiores de 18 anos, com prática instrumental em alguma orquestra ou grupos profissionalizantes, no mínimo 4 e no máximo 58 horas de prática semanal e frequência de 1 ano. $\mathrm{O}$ critério de exclusão foi não assinar o Termo de Consentimento Livre e Esclarecido (TCLE).

Foi realizada análise descritiva dos dados qualitativos por meio de frequência absoluta e relativa e dos dados quantitativos por mediana, percentis 25 e 75 e intervalo de confiança de 95\%, visto que os dados não apresentam distribuição normal (teste de Shapiro-Wilk, $\mathrm{p}<0,05)$. Adotou-se nível de confiança de $95 \%$. O software estatístico utilizado foi o Stata ${ }^{\circledR}$ versão 11.0

\section{RESULTADOS}

A análise estatística foi realizada de forma que agrupou as famílias de instrumentos em cordas, percussão e sopro.

$\mathrm{Na}$ avaliação das características dos músicos voluntários deste estudo, foi observada a mediana de idade para instrumentos de cordas de 22 anos, para instrumentos de percussão, de 22 anos e para instrumentos de sopro, de 24 anos (Tabela 1).

A maioria dessa amostra era do sexo masculino, sendo que dos instrumentos de cordas 23 (57,5\%) eram homens, dos instrumentos de percussão, oito $(88,9 \%)$ e dos instrumentos de sopro, $21(67,7 \%$ - Tabela 1$)$. 
Foi observado que, para todas as famílias de instrumentos, a metade ou mais apresentaram alguma dor causada pela prática instrumentista e mais da metade deles não faziam um acompanhamento médico, não apresentavam diagnósticos e não realizavam nenhum tratamento (Tabela 1 ).

Para a realização de atividades físicas, esta amostra mostrou-se ciente dos seus benefícios, sendo que 25 (62,5\%) dos que tocavam instrumentos de cordas, 5 (55,6\%) dos que tocavam instrumento de percussão e $17(54,8 \%)$ dos que tocavam instrumento de sopro praticavam algum exercício físico como natação, musculação, corrida, ciclismo, futebol, jiu-jítsu, ioga, dança, skate e basquete (Tabela 1).

A frequência de ensaios e intensidade durante a semana variava muito de uma orquestra a outra, principalmente quando uma apresentação estava próxima. Os músicos que tocavam instrumentos de sopro mostraram que passam maior tempo ensaiando, com uma mediana de 10 horas/semana, o que não se fez diferente na frequência de estudo, que foi de 15 horas/semana. Os instrumentos de cordas apresentaram a frequência de ensaios de 6 horas/semana e 10 horas/semana para a frequência de estudos. Já para percussão, não houve diferença significativa entre as frequências de ensaio e estudo, ambas foram de 5 horas/semana (Figuras 1 e 2).

$\mathrm{Na}$ avaliação do Questionário Nórdico de Sistemas Osteomusculares, em relação à presença de algum problema (como dor e formigamento/dormência) nos últimos 12 meses, as mãos, a região da coluna dorsal e a região da coluna lombar foram os locais que mais apresentaram queixas. Para todas essas regiões, houve uma prevalência de queixas para os músicos que tocavam os instrumentos de cordas. Dos 45 músicos que apresentaram queixas nas regiões das mãos, 25 (55,5\%) tocavam instrumentos de cordas; dos 41 músicos que apresentaram queixas na região da coluna dorsal, $23(56,1 \%)$ tocavam instrumentos de cordas; e dos 36 músicos que apresentaram queixas na região coluna lombar, 19 $(52,8 \%)$ tocavam instrumentos de cordas (Tabela 2$)$.

Foi observado que nos últimos 7 dias as regiões que mais apresentaram algum problema foram as regiões da coluna dorsal, com 25 músicos, e a região das mãos, com 22 músicos.
Tabela 1: Características sociodemográficas e sintomatologia relacionada ao tocar

\begin{tabular}{|c|c|c|c|}
\hline \multirow{2}{*}{ Variáveis } & Cordas & Percussão & Sopro \\
\hline & \multicolumn{3}{|c|}{ n (\%) } \\
\hline \multicolumn{4}{|l|}{ Sexo } \\
\hline Masculino & $23(57,5)$ & $8(88,9)$ & $21(67,7)$ \\
\hline Feminino & $17(42,5)$ & $1(11,1)$ & $10(32,3)$ \\
\hline \multicolumn{4}{|l|}{ Dor } \\
\hline Sim & $27(67,5)$ & $6(66,7)$ & $23(74,2)$ \\
\hline Não & $13(32,5)$ & $3(33,3)$ & $8(25,8)$ \\
\hline \multicolumn{4}{|c|}{ Acompanhamento médico } \\
\hline Sim & $2(5,0)$ & $2(22,2)$ & $3(9,7)$ \\
\hline Não & $38(95,0)$ & $7(77,8)$ & $28(90,3)$ \\
\hline \multicolumn{4}{|l|}{ Apresenta diagnóstico } \\
\hline Sim & $6(15,0)$ & $0(0)$ & $5(16,1)$ \\
\hline Não & $34(85,0)$ & $9(100)$ & $26(83,9)$ \\
\hline \multicolumn{4}{|l|}{ Tratamento* } \\
\hline Sim & $3(7,5)$ & $2(22,2)$ & $2(6,5)$ \\
\hline Não & $37(92,5)$ & $7(77,8)$ & $29(93,5)$ \\
\hline \multicolumn{4}{|l|}{ Atividade física } \\
\hline Sim & $25(62,5)$ & $5(55,6)$ & $17(54,8)$ \\
\hline Não & $15(37,5)$ & $4(44,4)$ & $14(45,2)$ \\
\hline \multicolumn{4}{|l|}{ Mediana (p.25 - p.75) } \\
\hline Idade (anos) & $22(18,0-27,5)$ & $22(19,0-27,0)$ & $24(19,0-30,0)$ \\
\hline $\begin{array}{l}\text { Frequência de ensaio } \\
\text { (horas/semana) }\end{array}$ & $6(5,0-10,0)$ & $5(2,0-8,0)$ & $10(6,0-14,0)$ \\
\hline $\begin{array}{l}\text { Frequência de estudo } \\
\text { (horas/semana) }\end{array}$ & $10(6,0-20,0)$ & $5(4,0-8,0)$ & $15(10,0-21,0)$ \\
\hline
\end{tabular}

p.25: percentil $25 \%$; p.75: percentil $75 \%$

*Medicamentoso, fisioterapia.

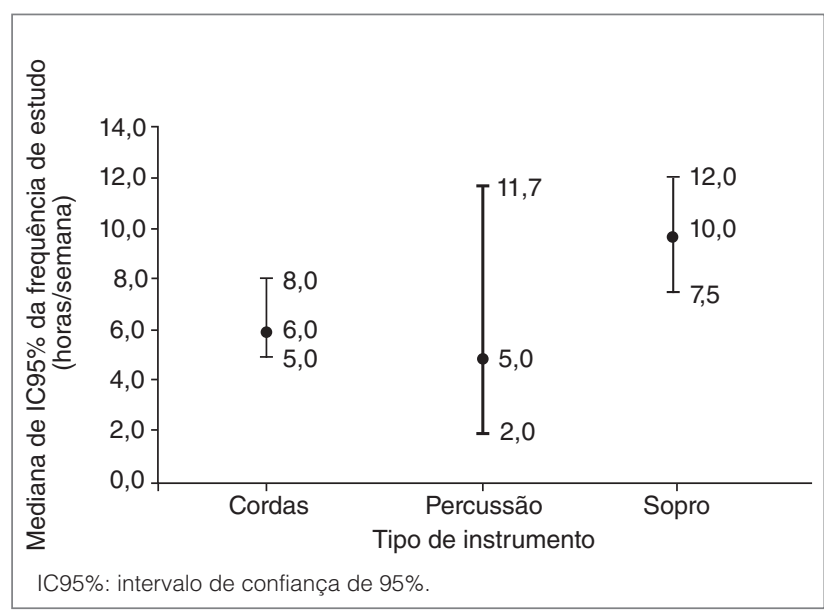

Figura 1: Valores de mediana e IC95\% da frequência de ensaio (em horas/semana) dos músicos pelo tipo de instrumento tocado (2014)

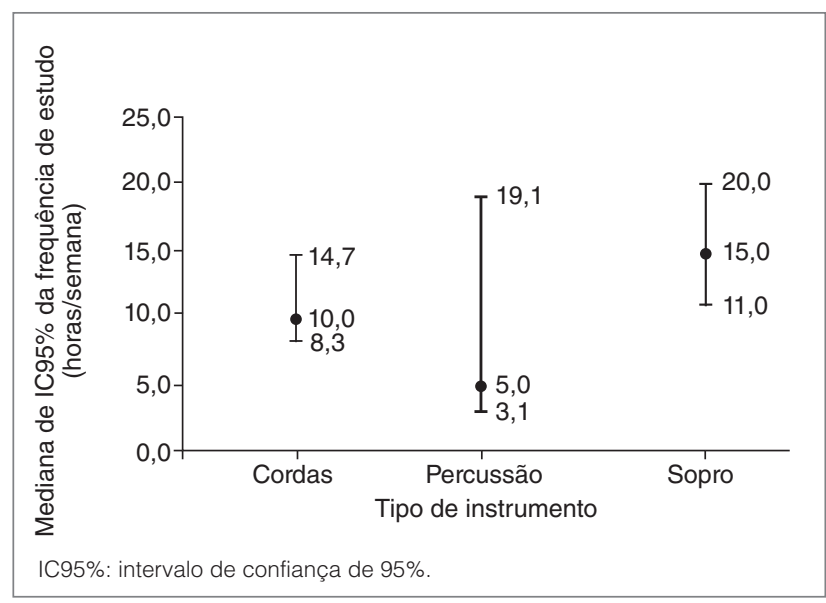

Figura 2: Valores de mediana e IC95\% da frequência de estudo (em horas/semana) dos músicos pelo tipo de instrumento tocado (2014) 
Houve prevalência de queixas dos músicos que tocavam instrumento de cordas: 14 (56,0\%) músicos para a região da coluna dorsal e 12 (54,9\%) para a região das mãos (Tabela 2).

$\mathrm{Na}$ presença de dor nos últimos 12 meses, em que houve impedimento em realizar atividades de vida diárias (por exemplo: trabalho, atividades domésticas e de lazer) por causa desses problemas, a mão foi o local quanto ao que mais músicos apresentaram queixas, com 8 no total, sendo que $5(62,5 \%)$ dos que tocavam instrumentos de cordas referiram impedimento. Outro local foi o joelho, em que seis músicos apresentaram queixas, sendo que $3(50,0 \%)$ tocavam instrumentos de cordas. Já quanto aos ombros e à região da coluna dorsal, cinco músicos apresentaram queixas, sendo que, para as duas regiões, as queixas maiores foram dos músicos que tocavam instrumentos de sopro, com $4(80,0 \%)$ para os ombros e 3 $(60,0 \%)$ para a região da coluna dorsal (Tabela 3$)$.

A procura por profissional da saúde nos últimos 12 meses para as determinadas regiões anatômicos foi baixa. As mãos novamente apresentaram prevalência de queixa, com dez músicos, porém notou-se que, tanto para os instrumentos de cordas quanto para os instrumentos de sopro, 4 músicos $(40,0 \%)$ procuraram assistência médica, enquanto para percussão apenas $2(20,0 \%)$. Para a região da coluna dorsal, oito músicos apresentaram queixas, porém a procura por

Tabela 2: Prevalência de sintomas (dor, formigamento/dormência) nos últimos 12 meses e presença de dor nos últimos 7 dias, pelo tipo de instrumento tocado (2014)

\begin{tabular}{|l|c|c|c|}
\hline Regiões anatômicas & Cordas & Percussão & Sopro \\
\hline Últimos 12 meses & \multicolumn{3}{|c|}{} \\
\hline Pescoço $(n=26)$ & $16(61,6)$ & $3(11,5)$ & $7(26,9)$ \\
\hline Ombro $(n=28)$ & $16(57,2)$ & $2(7,1)$ & $10(35,7)$ \\
\hline Região da coluna dorsal $(n=41)$ & $23(56,1)$ & $3(7,3)$ & $15(36,6)$ \\
\hline Cotovelo $(n=9)$ & $3(33,3)$ & $2(22,2)$ & $4(44,4)$ \\
\hline Mãos $(n=45)$ & $25(55,5)$ & $4(8,9)$ & $16(35,6)$ \\
\hline Região da coluna lombar $(n=36)$ & $19(52,8)$ & $3(8,3)$ & $14(38,9)$ \\
\hline Quadril $(n=9)$ & $5(55,6)$ & $1(11,1)$ & $3(33,3)$ \\
\hline Joelho $(n=12)$ & $7(58,3)$ & $2(16,7)$ & $3(25,0)$ \\
\hline Pés $(n=20)$ & $13(65,0)$ & $2(10,0)$ & $5(25,0)$ \\
\hline Últimos 7 dias & & & \\
\hline Pescoço $(n=15)$ & $8(53,3)$ & $0(0)$ & $7(46,7)$ \\
\hline Ombro $(n=17)$ & $9(52,9)$ & $1(5,9)$ & $7(41,2)$ \\
\hline Região da coluna dorsal $(n=25)$ & $14(56,0)$ & $1(4,0)$ & $10(40,0)$ \\
\hline Cotovelo $(n=6)$ & $2(33,3)$ & $1(16,7)$ & $3(50,0)$ \\
\hline Mãos $(n=22)$ & $12(54,6)$ & $3(13,6)$ & $7(31,8)$ \\
\hline Região coluna lombar $(n=17)$ & $7(41,2)$ & $1(5,9)$ & $9(52,9)$ \\
\hline Quadril $(n=3)$ & $2(66,7)$ & $0(0)$ & $1(33,3)$ \\
\hline Joelho $(n=7)$ & $5(71,4)$ & $1(14,3)$ & $2(14,3)$ \\
\hline Pés $(n=12)$ & $8(66,7)$ & $1(8,3)$ & $3(25,0)$ \\
\hline
\end{tabular}

assistência médica prevaleceu no grupo dos que tocavam instrumentos de cordas, com $5(62,5 \%)$ músicos. Já para os ombros e a região da coluna lombar, 7 músicos apresentaram queixas e, para as 2 regiões, $4(57,1 \%)$ músicos que tocavam instrumentos de sopro procuraram assistência médica (Tabela 4).

\section{DISCUSSÃO}

Este estudo observou que a dor é um sintoma muito frequente nesta população. Cinquenta e seis músicos relataram sentir dor causada pela prática instrumental, tendo como principais regiões coluna dorsal, seguida por punhos/ mãos, região da coluna lombar e ombro, pescoço, tornozelos/ pés, joelhos, cotovelos e, por fim, quadril/coxa. Em um estudo realizado com 46 músicos profissionais do Estado de Goiás e do Distrito Federal, em relação à dor, 27 músicos relataram ter atualmente desconforto, sendo os locais mais citados as mãos, os braços, os ombros e a coluna cervical e lombar ${ }^{11}$.

Tabela 3: Prevalência de impedimento ao realizar atividades de vida diária* devido a problemas nos últimos 12 meses pelo tipo de instrumento tocado (2014)

\begin{tabular}{|l|c|c|c|}
\hline \multirow{2}{*}{ Regiões anatômicas } & Cordas & Percussão & Sopro \\
\hline Pescoço $(n=3)$ & $2(66,7)$ & $0(0)$ & $1(33,3)$ \\
\hline Ombro $(n=5)$ & $1(20,0)$ & $0(0)$ & $4(80,0)$ \\
\hline Região da coluna dorsal "s" (n=5) & $2(40,0)$ & $0(0)$ & $3(60,0)$ \\
\hline Cotovelo $(n=2)$ & $1(50,0)$ & $0(0)$ & $1(50,0)$ \\
\hline Mãos $(n=8)$ & $5(62,5)$ & $1(12,5)$ & $2(25,0)$ \\
\hline Região da coluna lombar $(n=5)$ & $3(60,0)$ & $0(0)$ & $2(40,0)$ \\
\hline Quadril $(n=1)$ & $0(0)$ & $0(0)$ & $1(100)$ \\
\hline Joelho $(n=6)$ & $3(50,0)$ & $1(16,7)$ & $2(33,3)$ \\
\hline Pés $(n=4)$ & $3(75,0)$ & $0(0)$ & $1(25,0)$ \\
\hline
\end{tabular}

*Trabalho, atividades domésticas e de lazer.

Tabela 4: Prevalência de consulta a profissional da saúde* nos últimos 12 meses devido a algum problema pelo tipo de instrumento tocado (2014)

\begin{tabular}{|l|c|c|c|}
\hline \multirow{2}{*}{ Regiões anatômicas } & Cordas & Percussão & Sopro \\
\cline { 2 - 4 } & \multicolumn{3}{|c|}{$\mathbf{n}(\%)$} \\
\hline Pescoço $(n=6)$ & $3(50,0)$ & $0(0)$ & $3(50,0)$ \\
\hline Ombro $(n=7)$ & $3(42,9)$ & $0(0)$ & $4(57,1)$ \\
\hline Região da coluna dorsal $(n=8)$ & $5(62,5)$ & $0(0)$ & $3(37,5)$ \\
\hline Cotovelo $(n=3)$ & $1(33,3)$ & $0(0)$ & $2(66,7)$ \\
\hline Mãos $(n=10)$ & $4(40,0)$ & $2(20,0)$ & $4(40,0)$ \\
\hline Região da coluna lombar $(n=7)$ & $3(42,9)$ & $0(0)$ & $4(57,1)$ \\
\hline Quadril $(n=1)$ & $0(0)$ & $0(0)$ & $1(100)$ \\
\hline Joelho $(n=4)$ & $2(50,0)$ & $1(25,0)$ & $1(25,0)$ \\
\hline Pés $(n=3)$ & $2(66,7)$ & $0(0)$ & $1(33,3)$ \\
\hline
\end{tabular}

*Médico, fisioterapeuta. 
Observou-se, nessa amostra de músicos, um extenso número de horas dedicado ao instrumento tanto durante os ensaios quanto os estudos, o que pode justificar o número de músicos que relatam sentir dores durante e após a prática instrumental. Há, no instrumentista, uma busca incansável por uma técnica aperfeiçoada, o que exige longas horas de estudo, chegando inclusive à privação do convívio social, que podem aumentar o risco de lesão ocupacional ${ }^{11-13}$. Em estudo realizado sobre desordens ocupacionais em pacientes indicaram claramente que há uma relação entre os músicos, afirma-se que "a maioria do tempo de performance e a dor, sendo que, quando o tempo aumenta, a dor também aumenta"

A estrutura do instrumento é outro fator causador de problemas para os instrumentistas, pois o padrão postural adotado é assimétrico9. Dawson apud Alves afirma que características ergonômicas dos instrumentos e suas exigências biomecânicas específicas parecem determinar um papel quanto à etiologia, ou seja, à causa, à localização anatômica e à natureza dos problemas em músicos ${ }^{9}$.

$\mathrm{O}$ alongamento antecedendo a atividade é uma forma paliativa para o sintoma ${ }^{6,14}$. Estudos afirmam que os alongamentos diminuem o risco de lesão músculo-esquelética, aumentam o relaxamento muscular e a circulação sanguínea e melhoram a coordenação e as posturas estática e dinâmica. Além disso, aumentam a eficiência da performance, já que aumentam a flexibilidade muscular, mantêm a amplitude de movimento e aumentam a força da unidade músculo-tendínea, permitindo uma contração e fazendo com que a armazenagem de energia seja mais eficiente $\mathrm{6}^{6,15,16}$.

Um dado importante que podemos perceber é que apenas 5 músicos fazem acompanhamento médico, 11 apresentam diagnósticos e apenas 7 realizam algum tratamento. Dados relativamente baixos para o número de músicos que apresentou dor como sintoma pela prática instrumental. A falta de conhecimento e de acompanhamento do meio científico relacionado às questões de saúde dos músicos aliada com as problemáticas do medo em se perder oportunidades de um mercado restrito e até de perder o emprego, se afastados, fazem com que haja negligência em não se aceitar ou de se conviver com as dores e com o desconforto gerado pela prática do instrumento ${ }^{1}$.
Os movimentos que induzem o estresse podem ser classificados em dois tipos: movimentos isotônicos, que são movimentos mais sofisticados e rápidos, os que produzem sons; e movimentos isométricos, que servem para estabilizar e sustentar os instrumentos por grandes períodos ${ }^{8}$. Portanto, podemos observar que os locais que apresentam maior número de lesões em todo o Questionário Nórdico de Sintomas Osteomusculares foram os que realizam esses movimentos, como as mãos, que fazem os movimentos isotônicos, e os que fazem os movimentos isométricos, que são região da coluna dorsal e lombar, ombros e pescoço. Para que seja possível evitar o estresse desses músculos, o fortalecimento muscular, principalmente dos músculos estabilizadores do tronco e membros superiores, é essencial, fazendo com que a musculatura tenha maior resistência e força, exigindo menos esforço dela, visto que são regiões mais afetadas pela demanda do instrumento ${ }^{17}$.

Os resultados encontrados nos permitem concluir que as regiões das mãos e da coluna dorsal e lombar foram os locais mais afetados pela prática instrumental independente de a qual família o instrumento pertença, pois são áreas que estão mais suscetíveis aos movimentos repetitivos como as mãos ou as áreas de sustentação que, além de sustentar o peso do instrumento, devem manter a postura correta durante longos períodos.

Não foi possível analisar e relacionar a frequência das lesões osteomusculares dos músicos, pois a grande maioria não realizava acompanhamento médico e não apresentava diagnóstico clínico.

Com base nesses dados, devemos nos manter atentos a essas regiões anatômicas para que futuras lesões sejam prevenidas.

\section{AGRADECIMENTOS}

Aos maestros da Big Band da Fundação das Artes de São Caetano do Sul, Ogair Junior e da Orquestra Sinfônica Jovem da Fundação das Artes de São Caetano do Sul, Geraldo Oliviere; ao coordenador da escola Fundação das Artes de São Caetano do Sul, Daniel Volpin; a Regina Puerta Reijani, presidente da Associação Cultural Tocando a Vida; e a Isabella Reijani.

\section{REFERÊNCIAS}

1. Teixeira CS, Merino EAD, Lopes LF. A atividade do músico de orquestra: prática instrumental e desconforto corporal. Disponível em: http://boletimef.org/.../BoletimEF.org_Musico-de-orquestra-praticainstrumental-e-desconforto-corporal.pdf. Acesso em: 04 maio 2015.

2. Costa CP. Contribuições da ergonomia à saúde do músico: considerações sobre a dimensão física do fazer musical. Música Hodie. 2005;5(2):53-63.
3. Blum J. Medizinische Probleme bei Musikern. New York: Georg Thieme Verlag; 1995

4. Woellner SS, Martina DM, Kienen L. Distonia focal da mão em músicos: implicações para a reabilitação. Arq Catarin Med. 2013;42(3):82-8.

5. Fry HJ. Prevalence of overuse (injury) syndrome in Australian music schools. Br J Ind Med. 1987;44(1):35-40. 
6. Silva FC, Batista JC, Cruz IR, Deusdará FF, Soares WD. Músicos e o superuso do músculo esquelético. Col Pesq Educação Física. 2008;11(3):1981-4313.

7. Frank $A$, Mühlen CAV. Queixas musculoesqueléticas em músicos: prevalência e fatores de risco. Rev Bras Reumatol. 2007;47(3):188-96. http://dx.doi.org/10.1590/S0482-50042007000300008

8. Han-Sung L, Park HY, Yoon JO, Kim JS, Chun JM, Aminata IW, et al. Musculoskeletal problems in string players. Clin Orthop Surg. 2013;5(3):155-60.

http://dx.doi.org//10.4055/cios.2013.5.3.155

9. Alves CV. Padrões físicos inadequados na performance musical de estudantes de violino. Dissertação (Mestrado) - Universidade Federal de Minas Gerais, Belo Horizonte, 2008.

10. Trelha CS, Carvalho RP, Franco SS, Nakaoski T, Broza TP, Fábio TL, et al. Arte e saúde: frequência de sintomas músculo-esqueléticos em músicos da orquestra sinfônica da Universidade Estadual de Londrina. Semina: Ciênc Biol Saúde. 2004;25(1):65-72. http://dx.doi.org/10.5433/1679-0367.2004v25n1p65

11. Fragelli TBO, Günther IA. Relação entre dor e antecedentes de adoecimento físico ocupacional: um estudo entre músicos instrumentistas. Per Musi. 2008:(19):18-23. http://dx.doi.org/10.1590/S1517-75992009000100003
12. Subtil MML, Bonomo LMM. Avaliação fisioterapêutica nos músicos de uma orquestra filarmônica. Per Musi. 2012;(25):85-90. http://dx.doi.org/10.1590/S1517-75992012000100008

13. Chan C, Ackermann B. Evidence-informed physical therapy management of performance-related musculoskeletal disorders in musicians. Front Psychol. 2014;5:706. http://dx.doi.org/10.3389/fpsyg.2014.00706

14. Costa CP, Abrahão Jl. Quando o tocar dói: um olhar ergonômico sobre o fazer musical. Per Musi: Rev Acadêmica Música. 2004;(10):102.

15. Mazzoni CF, Vieira A, Guthier C, Perdigão D, Marçal MA. Avaliação da incidência de queixas músculo-esqueléticas em músicos instrumentistas de cordas friccionadas. Curitiba: ABERGO; 2006.

16. Fragelli TB, Gunther IA. Abordagem ecológica para avaliação dos determinantes de comportamento preventivos: inventário aplicado aos músicos. Per Musi. 2012;(25):73-84. http://dx.doi.org/10.1590/S1517-75992012000100007

17. Chan C, Driscoll T, Ackermann B. Development of a specific exercise programme for professional orchestral musicians. Inj Prev. 2013:1-7.

http://dx.doi.org/10.1136/injuryprev-2012-040608 